\title{
STRENGTH CALCULATIONS OF AN ELEMENT COMPENSATING CIRCUMFERENTIAL BACKLASH IN THE EXTERNAL GEAR PUMP
}

\author{
Piotr Osiński, Grzegorz Chruścielski \\ Wroclaw University of Technology, Faculty of Mechanical Engineering, Wroctaw, Poland \\ e-mail: piotr.osinski@pwr.edu.pl; grzegorz.chruscielski@pwr.edu.pl
}

\begin{abstract}
The aim of the article is to discuss the issue of strength of the circumferential backlash compensating beam in a high-efficiency gear pump. Three geometric versions of the compensating beam structure differing in the wrapping angle are analyzed. The mechanical model is solved assuming the curved beam model supported at the contact points between the beam and the gear teeth. The assumed mechanical structure is statically indeterminate. In order to determine the reactions in the supports and in the beam fixing, the Menabrei and Castigliano theorems are used. Based on analytical calculation results, the cause of compensation structure damage during experimental tests of prototype units is identified and the most favorable variant of beam structure, from the mechanical strength point of view, is determined.
\end{abstract}

Keywords: external gear pump, strength calculation, circumferential backlash compensation

\section{Introduction}

The efficiency of a gear pump is to a large extent determined by clearances between the gears and the elements limiting the displacement chamber volume of the pump (Chrobot et al., 1997; Judin, 1958; Kollek, 1996, 2004; Osiński et al., 2013; Osiński and Kollek, 2007; Ragunathan and Manoharan, 2012; Vacca and Guidetti, 2011; Wang et al., 2011). Two types of clearance, i.e. radial clearance and frontal clearance, are distinguished. The former is also referred to as circumferential clearance (backlash). The circumferential gap is formed by the surface of casing concavities and that of the cylinder with the radius of the addendum circle of toothed displacement elements. The gap is not constant along the whole circumference the gears often move within the bearing slackness limits towards the suction space. In conventional pumps without radial backlash compensation, the circumferential gap assumes the shape of a crescent widening towards the delivery side. In such a pump design, the gap ranges from 0.01 to $0.3 \mathrm{~mm}$. The circumferential clearance is a gap with one fixed wall and one movable wall moving in the direction opposite to that of the pressure drop. This is an advantageous configuration as a result of the rotational motion of the gear the liquid is lifted from the suction space to the delivery space, thereby reducing the leakages due to the pressure difference between the gap ends (Singal et al., 2009; Stryczek, 1995).

Frontal clearances have the shape of a ring limited by the diameter of the dedendum circle and that of the gear shaft. Most of the leakages are used to cool and lubricate the bearings. After they pass through the bearings, the leakages are directed via special grooves to the suction chamber, but some of the volume losses pass directly through the gap into the suction space. The frontal clearance values are by one order of magnitude lower than the radial clearance values. In typical pumps, frontal clearances are in a range of 0.01-0.05 $\mathrm{mm}$. The recommended clearance also depends on the pump size. Lower values are recommended for units with a lower specific output. 
Nowadays backlash compensation is used to improve the efficiency of gear pumps (Kollek and Radziwanowska, 2015). In addition, this treatment contributes to better running in of the interacting parts and maintenance of a constant gap despite the wear of the parts. In the currently produced pumps, mainly the axial backlash compensation is carried out. However, if higher efficiency is required, it is necessary to compensate also the circumferential gap. The latter can be compensated in two ways. One way consists in compensating backlash locally along a short distance. It is further referred to as radial backlash compensation (Fig. 1a). Another method, developed by the authors, consists in ensuring a constant gap along the whole circumference (Osiński, 2012a,b; Wiczkowski, 2012).The method is called circumferential backlash compensation (Fig. 1c).
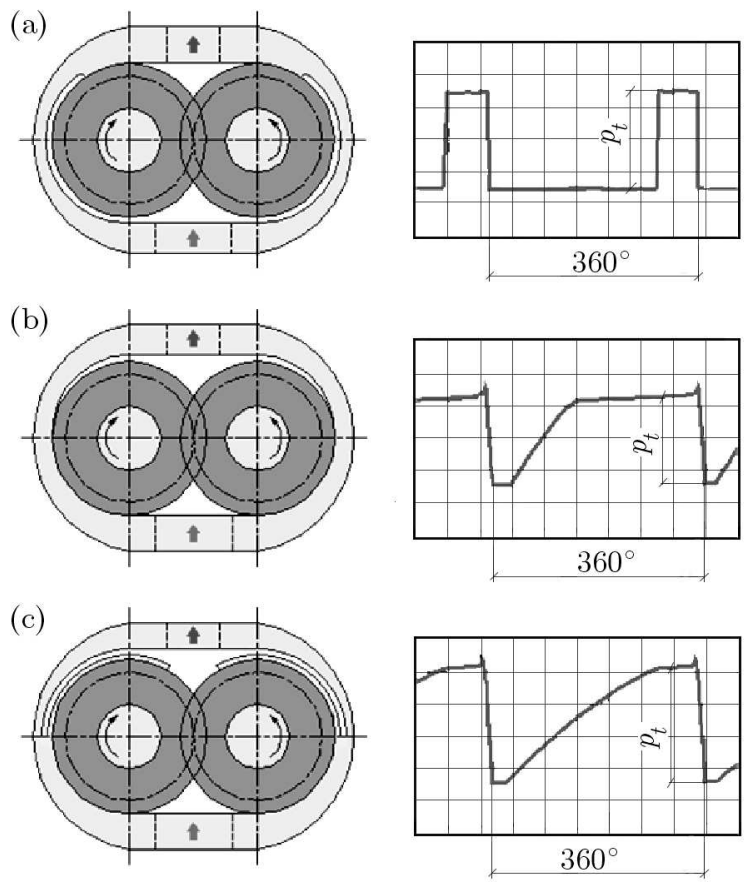

Fig. 1. Schematic showing the way of sealing gears along circumference plus graph of circumferential pressure measured in the gear root: (a) pump with radial backlash compensation, (b) pump with axial backlash compensation, (c) pump with circumferential backlash compensation (Osiński et al., 2012a)

The influence of the applied compensation on the overall efficiency of the pump is shown in Fig. 2. The comparative diagram is based on specifications found in the manufacturer catalogues of Bosch, Casappa, Marzocchi, Hamworthy, Hidroirma, Orsta, Parker, PZL-Hydral, Rexroth, WPH, VPS and on the authors' own studies of prototype pumps with circumferential backlash compensation (Osiński, 2013).

It appears from the diagram that the application of different methods of backlash compensation considerably increases the efficiency and working pressure of gear pumps. The currently produced pumps reach working pressures as high as $32 \mathrm{MPa}$. The innovative circumferential backlash compensation method enables one to increase the pressures by nearly $20 \%$, i.e. to the level of $40 \mathrm{MPa}$. The increasing of the internal tightness also makes it possible to increase the total efficiency by about $5 \%$ on average.

\section{Circumferential compensation structure}

There are three versions of the displacement pump structure with a compensating pressure chamber (Osiński, 2013; Osiński et al., 2012), differing in the design of the compensating chamber 


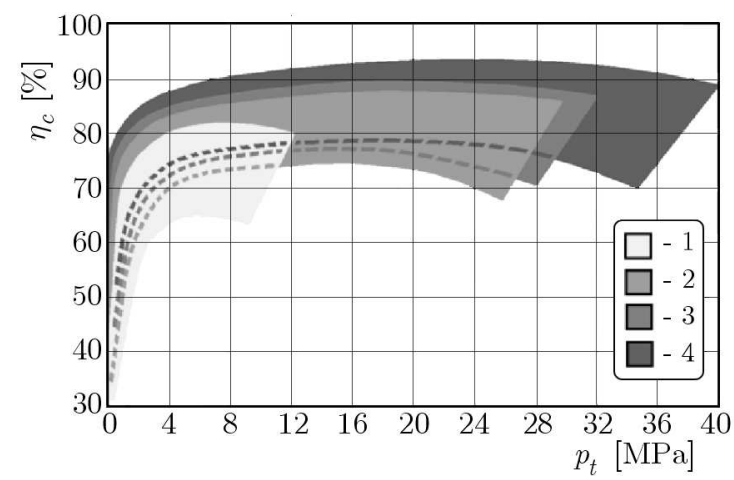

Fig. 2. Comparison of total efficiency $\eta_{c}$ of gear pumps with regard to forcing pressure $p_{t}$ and backlash compensation (based on catalogues of major manufacturers + own research): 1 - without compensation, 2 - with axial compensation, 3 - with axial and radial compensation, 4 - with axial and circumferential compensation

(Fig. 3). In this pump, two interacting gears perform rotations in the directions marked in Fig. 3, forcing the working liquid (oil) from the suction chamber on the left side of the pump (Fig. 3) through the inter tooth spaces into the delivery chamber on the right side of the pump.
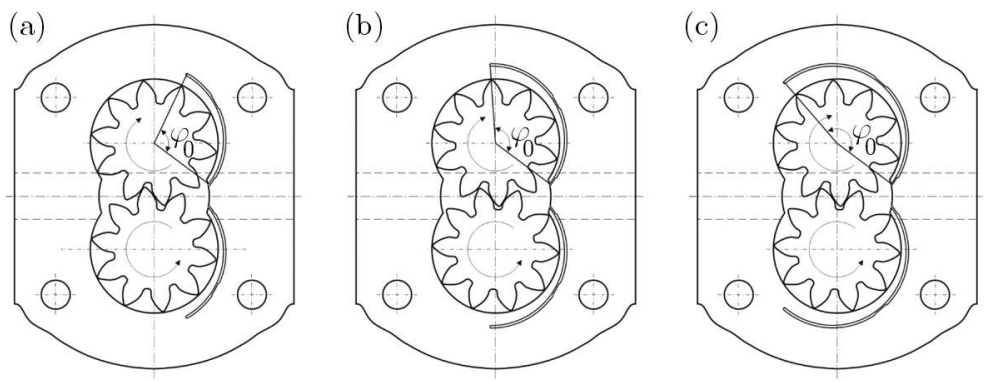

Fig. 3. Schematic of the displacement pump with different compensating beam designs: (a) beam with a wrapping angle $\varphi_{0}=102^{\circ}$, (b) $\varphi_{0}=132^{\circ}$, (c) $\varphi_{0}=169^{\circ}$

Experimental studies of prototypes of such pumps have shown that during operation under heavy loads (at pressures $p$ above $20 \mathrm{MPa}$ ) the beam closing the compensating pressure chamber is susceptible to failure in the place of its fixing because of too small beam thickness. The aim of the calculations presented in this paper is to determine (from the strength condition) the minimum thickness $h$ in the fixed cross section of the beam ensuring that the stresses in this cross section are carried.

Three geometric versions of the compensating beam structure have been designed. The most optimal version will be selected on the basis of theoretical calculations and experiments. The versions differ in the beam length, i.e. its wrapping angle, and so in the number of teeth interacting with the beam. For the statical analysis, a fixed gear position in which one of the teeth is in contact with the beam fixing cross section is assumed in each of the cases. The versions include:

a) a beam with a wrapping angle $\varphi_{0}=102^{\circ}$, interacting with three teeth of the gear, one of which is in contact with the beam fixing cross section (Fig. 3a);

b) a beam with a wrapping angle $\varphi_{0}=132^{\circ}$, interacting with four teeth of the gear, one of which is in contact with the beam fixing cross section (Fig. 3b);

c) a beam with a wrapping angle $\varphi_{0}=169^{\circ}$, interacting with five teeth of the gear, one of which is in contact with the beam fixing cross section (Fig. 3c). 


\section{Static calculations for the pressure chamber beam}

3.1. Beam with a wrapping angle $\varphi_{0}=102^{\circ}$

\subsubsection{Beam geometry and loading diagram}

During the operation of the pump, the compensating chamber beam is loaded from the outside with compensating pressure $p_{2}$ constant along the whole length of the beam, and from the inside with working pressure $p_{1}$ (Fig. 4a). The pressure $p_{1}$ decreases in the successive intertooth spaces from the initial value $p_{1 p}=p_{2}$ at the inlet to the compensating chamber up to end value $p_{1 k}=0.5 p_{2}$ in the tooth space at the beam fixing. Thus the pressure difference $\Delta p=p_{2}-p_{1 k}$ constitutes a linearly variable continuous load $q(\varphi)$ for the beam, whose initial value is $q(\varphi=0)=0$ and its end value is $q\left(\varphi=2 \varphi_{1}+\alpha\right)=q_{0}=\Delta p b$, where $b$ is the beam width (Fig. 4b). Then the beam load can be reduced to a flat system.
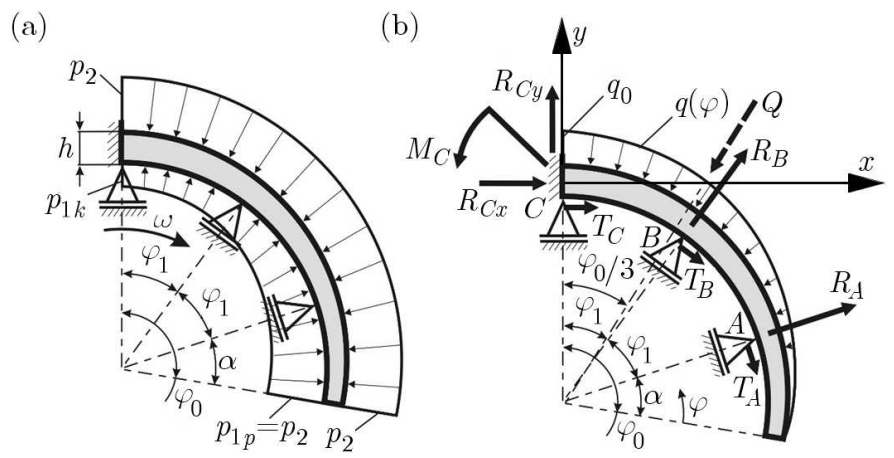

Fig. 4. (a) Static diagram of the beam with the wrapping angle of $102^{\circ}$, loaded with working pressure $p_{1}$ and compensating pressure $p_{2}$, (b) diagram after introduction of continuous load replacing the action of pressures $p_{1}$ and $p_{2}$

As a result of the difference between the pressure $p_{2}$ and $p_{1}$, the beam comes into contact with the pump gear teeth, constituting movable supports of the beam, in points $A$ and $B$ (Fig. $2 \mathrm{~b}$ ). Respective reactions $R_{A}$ and $R_{B}$ and friction forces $T_{A}$ and $T_{B}$, whose sense is consistent with the direction of the rotational motion of the pump gear, occur in the supports. Two reactions: $R_{C x}$ and $R_{C y}$ and fixing moment $M_{C}$ occur in the beam fixing place (point $C$ ). The directions of reactions $R_{C x}$ and $R_{C y}$ correspond to the adopted flat reference system (xy) whose origin is in the centre of gravity of the fixed cross section and which is connected with the normal and tangent direction of this cross section (Fig. 4b).

The angles $\varphi_{1}$ (marked in Fig. 4) between the central surfaces of the teeth amount to $\varphi_{1}=360^{\circ} / 10=36^{\circ}$ (the pump gears have 10 teeth) while the complementary angle between point $A$ and the beginning of the beam amounts to $\alpha=30^{\circ}$. The beam width (in the direction perpendicular to the load surface) is constant and amounts to $b=26.3 \mathrm{~mm}$.

\subsubsection{Solutions for beam static load system}

For the assumed beam loading diagram (Fig. 4b), the static equilibrium equations have the form

$$
\begin{aligned}
& \sum P_{x}=R_{C x}-Q_{x}+R_{B x}+R_{A x}+T_{C}+T_{B x}+T_{A x}=0 \\
& \sum P_{y}=R_{C y}-Q_{y}+R_{B y}+R_{A y}-T_{B y}-T_{A y}=0 \\
& \sum M^{C}=M_{C}+R_{B} r \sin \varphi_{1}+R_{A} r \sin 2 \varphi_{1}-T_{B}\left(r-r \cos \varphi_{1}\right)-T_{A}\left(r-r \cos 2 \varphi_{1}\right) \\
& \quad-Q r \sin \left(\varphi_{c} / 3\right)=0
\end{aligned}
$$


The equations include the reaction force and friction force components amounting to: $R_{A x}=R_{A} \sin 2 \varphi_{1}, R_{A y}=R_{A} \cos 2 \varphi_{1}, R_{B x}=R_{B} \sin \varphi, R_{B y}=R_{B} \cos \varphi_{1}, T_{A x}=T_{A} \cos 2 \varphi_{1}$, $T_{A y}=T_{A} \sin 2 \varphi_{1}, T_{B x}=T_{B} \cos \varphi_{1}, T_{B y}=T_{B} \sin \varphi_{1}$. Moreover, the action of continuous load $q(\varphi)$ has been replaced with the concentrated force $Q$ applied to the point corresponding to angle $\varphi=2 / 3 \varphi_{0}$, where: $\varphi_{0}=\left(2 \varphi_{1}+\alpha\right)$ is the maximum angle $\varphi$ value (for the whole beam span). Then the value of force $Q$ can be calculated from the formula

$$
Q=\frac{1}{2} q_{0} r \varphi_{0}
$$

and the force components for the axes $x$ and $y$ amount to $Q_{x}=Q \sin \left(\varphi_{0} / 3\right)$ and $Q_{y}=Q \cos \left(\varphi_{0} / 3\right)$.

It appears from Eqs. (3.1) that the analyzed static system is a double hyperstatic system. The Menabrei energy method, according to which the derivative of the system elastic energy relative to the hyperstatic reaction amounts to zero (Zakrzewski and Zawadzki, 1983; Niezgodziński and Niezgodziński, 1996; Dyląg et al., 1999), will be used to determine reactions in the supports and in the beam fixing.

The bending moment equations and their derivatives over hyperstatic reactions $R_{A}$ and $R_{B}$ depending on the angle $\varphi$ for particular beam intervals are as follows:

- interval I $(0<\varphi \leqslant \alpha)$

$$
M_{g}^{I}(\varphi)=-\frac{q r^{2} \varphi^{2}}{2 \varphi_{0}} \sin \frac{\varphi}{3} \quad \frac{\partial M_{g}^{I}}{\partial R_{A}}=0 \quad \frac{\partial M_{g}^{I}}{\partial R_{B}}=0
$$

— interval II $\left(\alpha<\varphi \leqslant \alpha_{2}=\alpha+\varphi_{1}\right)$

$$
\begin{aligned}
& M_{g}^{I I}(\varphi)=-\frac{q r^{2} \varphi^{2}}{2 \varphi_{0}} \sin \frac{\varphi}{3}+R_{A} r \sin (\varphi-\alpha)-T_{A}[r-r \cos (\varphi-\alpha)] \\
& \frac{\partial M_{g}^{I I}}{\partial R_{A}}=r \sin (\varphi-\alpha) \quad \frac{\partial M_{g}^{I}}{\partial R_{B}}=0
\end{aligned}
$$

— interval III $\left(\alpha_{2}<\varphi \leqslant \varphi_{0}\right)$

$$
\begin{aligned}
& M_{g}^{I I I}(\varphi)=-\frac{q r^{2} \varphi^{2}}{2 \varphi_{0}} \sin \frac{\varphi}{3}+R_{A} r \sin (\varphi-\alpha)-T_{A}[r-r \cos (\varphi-\alpha)] \\
& \quad+R_{B} r \sin \left(\varphi-\alpha_{2}\right)-T_{B}\left[r-r \cos \left(\varphi-\alpha_{2}\right)\right] \\
& \frac{\partial M_{g}^{I I I}}{\partial R_{A}}=r \sin (\varphi-\alpha) \quad \frac{\partial M_{g}^{I I}}{\partial R_{B}}=r \sin \left(\varphi-\alpha_{2}\right)
\end{aligned}
$$

In the above equations, the expression for the bending moment produced by continuous load $q(\varphi)=q_{0} \varphi / \varphi_{0}$ takes into account equation $(3.2): Q(\varphi)=0.5 r \varphi q(\varphi)=q_{0} r \varphi^{2} /\left(2 \varphi_{0}\right)$, assuming that for any cross section defined by angle $\varphi$ the substitute force $Q(\varphi)$ is applied to the point situated relative to this cross section at angle $\varphi / 3: M_{Q(\varphi)}=-Q(\varphi) r \sin (\varphi / 3)=$ $\left[-q r^{2} \varphi^{2} /\left(2 \varphi_{0}\right)\right] \sin (\varphi / 3)$. Moreover, in order to simplify the notation, the angle $\alpha_{2}=\alpha+\varphi_{1}$ is introduced for determination of the range of variation of angle $\varphi$ in intervals II and III.

According to the Menabrei theorem, hyperstatic reactions $R_{A}$ and $R_{B}$ can be calculated from the following system of equations 


$$
\begin{aligned}
& \frac{\partial V}{\partial R_{A}}=\left[\int_{\alpha}^{\alpha_{2}}\left(-\frac{q_{C} r^{2} \varphi^{2}}{2 \varphi_{0}} \sin \frac{\varphi}{3}+R_{A} r \sin (\varphi-\alpha)-T_{A} r[1-\cos (\varphi-\alpha))[r \sin (\varphi-\alpha)] d \varphi\right]\right. \\
& \quad+\frac{1}{E I}\left[\int _ { \alpha _ { 2 } } ^ { \varphi _ { C } } \left(-\frac{q_{C} r^{2} \varphi^{2}}{2 \varphi_{0}} \sin \frac{\varphi}{3}+R_{A} r \sin (\varphi-\alpha)-T_{A} r[1-\cos (\varphi-\alpha)]\right.\right. \\
& \left.\left.\quad+R_{B} r \sin \left(\varphi-\alpha_{2}\right)-T_{B} r\left[1-\cos \left(\varphi-\alpha_{2}\right)\right]\right)[r \sin (\varphi-\alpha)] d \varphi\right]=0 \\
& \frac{\partial V}{\partial R_{B}}=\frac{1}{E I}\left[\int _ { \alpha _ { 2 } } ^ { \varphi _ { 0 } } \left(-\frac{q_{C} r^{2} \varphi^{2}}{2 \varphi_{0}} \sin \frac{\varphi}{3}+R_{A} r \sin (\varphi-\alpha)-T_{A} r[1-\cos (\varphi-\alpha)]\right.\right. \\
& \left.\left.\quad+R_{B} r \sin \left(\varphi-\alpha_{2}\right)-T_{B} r\left[1-\cos \left(\varphi-\alpha_{2}\right)\right]\right)\left[r \sin \left(\varphi-\alpha_{2}\right)\right] d \varphi\right]=0
\end{aligned}
$$

Having solved the system of equations (3.6), one can calculate reactions $R_{A}$ and $R_{B}$

$$
\begin{aligned}
& R_{A}=0.2316 \Delta p b r+0.1915 T_{A}-0.0283 T_{B}=2376.7 \mathrm{~N} \\
& R_{B}=0.3870 \Delta p b r+0.8494 T_{A}+0.2997 T_{B}=3978.1 \mathrm{~N}
\end{aligned}
$$

Then using static equilibrium equations (3.1)one can calculate the reactions in the beam fixing

$$
\begin{aligned}
& R_{C x}=Q_{C x}-R_{B x}-R_{A x}-T_{C}-T_{B x}-T_{A x}=490.4 \mathrm{~N} \\
& R_{C y}=Q_{C y}-R_{B y}-R_{A y}+T_{B y}+T_{A y}=3628.1 \mathrm{~N} \\
& M_{C}=Q_{r} \sin \varphi_{0} 3-R_{B} r \sin \varphi_{1}-R_{A} r \sin 2 \varphi_{1}+T_{B}\left(r-r \cos \varphi_{1}\right) \\
& \quad+T_{A}\left(r-r \cos 2 \varphi_{1}\right)=13.35 \mathrm{~N}
\end{aligned}
$$

\subsection{Beam with the wrapping angle $\varphi_{0}=132^{\circ}$}

\subsubsection{Beam geometry and loading diagram}

The loading diagram for the beam with the wrapping angle $\varphi_{0}=132^{\circ}$ is shown in Fig. 5a, while its modified version (having pressures replaced with the continuous load) is shown in Fig. 3b. In the latter version, the beam interacts with four teeth of the gear and the working pressure $p_{1}$ decreases from the initial value $p_{1 p}=p_{2}$ for $\varphi=0$ up to end value $p_{1 k}=0.33 p_{2}$ for $\varphi=\varphi_{0}$. The continuous load acting on the beam, arising due to the pressure difference, and the resultant substitute force $Q$ is defined the same as for the beam with the wrapping angle $102^{\circ}$. Besides the continuous load also the friction forces $T_{A}, T_{B}, T_{C}, T_{D}$, reactions in the supports (at the contact with the pump teeth) $R_{A}, R_{B}, R_{C}$ and the reactions in the fixing $R_{D x}, R_{D y}, M_{D}$ (Fig. 5b) act on the beam.

\subsubsection{Solution for beam load static system}

For the loading diagram shown in Fig. 5b, the static equilibrium equations have the form

$$
\begin{aligned}
& \sum P_{x}=R_{D x}+R_{C x}+R_{B x}+R_{A x}+T_{D}+T_{C x}+T_{B x}-T_{A x}-Q_{x}=0 \\
& \sum P_{y}=R_{D y}+R_{C y}+R_{B y}-R_{A y}-T_{C y}-T_{B y}-T_{A y}-Q_{y}=0 \\
& \sum M^{D}=M_{D}+R_{C r} \sin \varphi_{1}+R_{B} r \sin 2 \varphi_{1}+R_{A} r \cos \frac{\varphi_{1}}{2}-T_{C}\left(r-r \cos \varphi_{1}\right) \\
& -T_{B}\left(r-r \cos 2 \varphi_{1}\right)-T_{A}\left(r+r \sin \frac{\varphi_{1}}{2}\right)-Q r \sin \frac{\varphi_{0}}{3}=0
\end{aligned}
$$


(a)

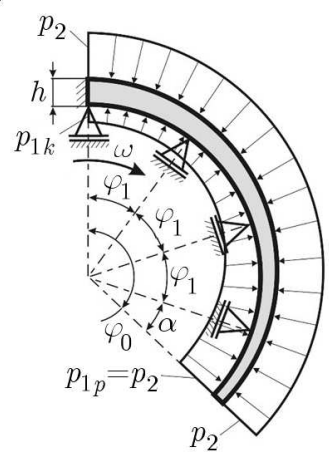

(b)

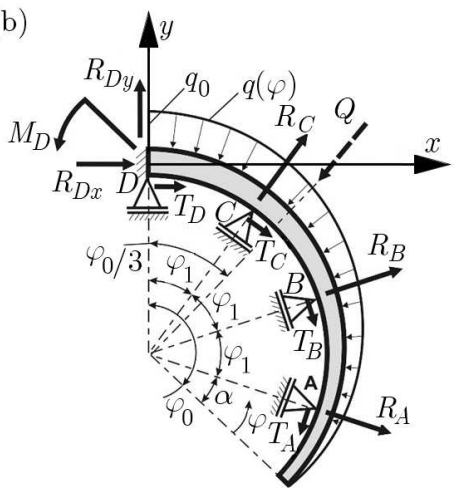

Fig. 5. (a) Static diagram of the beam with the wrapping angle of $132^{\circ}$, loaded with working pressure $p_{1}$ and compensating pressure $p_{2}$, (b) diagram after introduction of continuous load replacing the action of pressures $p_{1}$ and $p_{2}$

The equilibrium equations include the reaction and friction force components amounting to: $R_{A x}=R_{A} \cos \left(\varphi_{1} / 2\right), R_{A y}=R_{A} \sin \left(\varphi_{1} / 2\right), R_{B x}=R_{B} \sin 2 \varphi_{1}, R_{B y}=R_{B} \cos 2 \varphi_{1}$, $R_{C x}=R_{C} \sin \varphi_{1}, R_{C y}=R_{C} \cos \varphi_{1}, T_{A x}=T_{A} \sin \left(\varphi_{1} / 2\right), T_{A y}=T_{A} \cos \left(\varphi_{1} / 2\right), T_{B x}=T_{B} \cos 2 \varphi_{1}$, $T_{B y}=T_{B} \sin 2 \varphi_{1}, T_{C x}=T_{C} \cos \varphi_{1}, T_{C y}=T_{C} \sin \varphi_{1}, Q_{x}=Q \sin \left(\varphi_{0} / 3\right), Q_{y}=Q \cos \left(\varphi_{0} / 3\right)$.

Since the considered beam is a triple hyperstatic system, in order to calculate the reactions occurring in the supports and in the beam fixing one should formulate three equations based on the Menabrei method. Assuming $R_{A}, R_{B}$ and $R_{C}$ as hyperstatic reactions occurring in the supports, the equations become

$$
\begin{aligned}
& \frac{\partial V}{\partial R_{A}}=\frac{1}{E I}\left[\int_{\alpha}^{\alpha_{2}}\left(-\frac{q_{0} r^{2} \varphi^{2}}{2 \varphi_{0}} \sin \frac{\varphi}{3}+R_{A} r \sin (\varphi-\alpha)-T_{A} r[1-\cos (\varphi-\alpha)]\right)[r \sin (\varphi-\alpha)] d \varphi\right] \\
& +\frac{1}{E I}\left[\int _ { \alpha _ { 2 } } ^ { \alpha _ { 3 } } \left(-\frac{q_{0} r^{2} \varphi^{2}}{2 \varphi_{0}} \sin \frac{\varphi}{3}+R_{A} r \sin (\varphi-\alpha)-T_{A} r[1-\cos (\varphi-\alpha)]\right.\right. \\
& \left.\left.+R_{B} r \sin \left(\varphi-\alpha_{2}\right)-T_{B} r\left[1-\cos \left(\varphi-\alpha_{2}\right)\right]\right)[r \sin (\varphi-\alpha)] d \varphi\right] \\
& \quad+\frac{1}{E I}\left[\int _ { \alpha _ { 3 } } ^ { \varphi _ { 0 } } \left(-\frac{q_{0} r^{2} \varphi^{2}}{2 \varphi_{C}} \sin \frac{\varphi}{3}+R_{A} r \sin (\varphi-\alpha)-T_{A} r[1-\cos (\varphi-\alpha)]+R_{B} r \sin \left(\varphi-\alpha_{2}\right)\right.\right. \\
& \left.\left.\quad-T_{B} r\left[1-\cos \left(\varphi-\alpha_{2}\right)\right]+R_{C} r \sin \left(\varphi-\alpha_{3}\right)-T_{C} r\left[1-\cos \left(\varphi-\alpha_{3}\right)\right]\right)[r \sin (\varphi-\alpha)] d \varphi\right]=0 \\
& \frac{\partial V}{\partial R_{B}}=\frac{1}{E I}\left[\int _ { \alpha _ { 2 } } ^ { \alpha _ { 3 } } \left(-\frac{q_{0} r^{2} \varphi^{2}}{2 \varphi_{0}} \sin \frac{\varphi}{3}+R_{A} r \sin (\varphi-\alpha)-T_{A} r[1-\cos (\varphi-\alpha)]\right.\right. \\
& \left.\quad+R_{B} r\left[1-\sin \left(\varphi-\alpha_{2}\right)-T_{B} r\left[1-\cos \left(\varphi-\alpha_{2}\right)\right]\right)\left[r \sin \left(\varphi-\alpha_{2}\right)\right] d \varphi\right] \\
& \quad \frac{1}{E I}\left[\int _ { \alpha _ { 3 } } ^ { \varphi _ { 0 } } \left(-\frac{q_{0} r^{2} \varphi^{2}}{2 \varphi_{C}} \sin \frac{\varphi}{3}+R_{A} r \sin (\varphi-\alpha)-T_{A} r[1-\cos (\varphi-\alpha)]+R_{B} r \sin \left(\varphi-\alpha_{2}\right)\right.\right. \\
& \left.\left.\quad R_{C} r \sin \left(\varphi-\alpha_{3}\right)-T_{C} r\left[1-\cos \left(\varphi-\alpha_{3}\right)\right]\right)\left[r \sin \left(\varphi-\alpha_{2}\right)\right] d \varphi\right]=0
\end{aligned}
$$




$$
\begin{aligned}
& \frac{\partial V}{\partial R_{C}}=\frac{1}{E I}\left[\int _ { \alpha _ { 3 } } ^ { \varphi _ { 0 } } \left(-\frac{q_{0} r^{2} \varphi^{2}}{2 \varphi_{0}} \sin \frac{\varphi}{3}+R_{A} r \sin (\varphi-\alpha)-T_{A} r[1-\cos (\varphi-\alpha)]+R_{B} r \sin \left(\varphi-\alpha_{2}\right)\right.\right. \\
& \left.\left.\quad-T_{B} r\left[1-\cos \left(\varphi-\alpha_{2}\right)\right]+R_{C} r \sin \left(\varphi-\alpha_{3}\right)-T_{C} r\left[1-\cos \left(\varphi-\alpha_{3}\right)\right]\right)\left[r \sin \left(\varphi-\alpha_{3}\right)\right] d \varphi\right]=0
\end{aligned}
$$

Having solved equations (3.10), one can calculate reactions $R_{A}, R_{B}$ and $R_{C}$

$$
\begin{aligned}
& R_{A}=0.1103 \Delta p b r+0.2522 T_{A}-0.0259 T_{B}+0.0067 T_{C}=1510.2 \mathrm{~N} \\
& R_{B}=0.3164 \Delta p b r+0.7156 T_{A}-0.3009 T_{B}-0.0369 T_{C}=4334.5 \mathrm{~N} \\
& R_{C}=0.5946 \Delta p b r+0.6019 T_{A}-0.6787 T_{B}+0.3009 T_{C}=8144.1 \mathrm{~N}
\end{aligned}
$$

Finally, using static equilibrium equations (3.9), one gets the values of reactions in the beam fixing

$$
\begin{aligned}
& R_{D x}=Q_{x}-R_{C x}-R_{B x}-R_{A x}-T_{D}-T_{C x}-T_{B x}+T_{A x}=583.8 \mathrm{~N} \\
& R_{D y}=Q_{y}-R_{C y}-R_{B y}+R_{A y}+T_{C y}+T_{B y}+T_{A y}=3889.9 \mathrm{~N} \\
& M_{D}=Q r \sin \frac{\varphi_{0}}{3}-R_{C} r \sin \varphi_{1}-R_{B} r \sin 2 \varphi_{1}-R_{A} r \cos \frac{\varphi_{1}}{2}+T_{C}\left(r-r \cos \varphi_{1}\right) \\
& \quad+T_{B}\left(r-r \cos 2 \varphi_{1}\right)+T_{A}\left(r+r \sin \frac{\varphi_{1}}{2}\right)=15.98 \mathrm{~N}
\end{aligned}
$$

\subsection{Beam with the wrapping angle $\varphi_{0}=169^{\circ}$}

\subsubsection{Beam geometry and loading diagram}

Figures $6 \mathrm{a}$ and $6 \mathrm{~b}$ show the loading diagram and the diagram which takes into account the replacement of pressures $p_{1}$ and $p_{2}$ (acting on both sides of the beam) with continuous load $q$ for the pump with the compensating chamber with the wrapping angle $\varphi_{0}=169^{\circ}$.

(a)

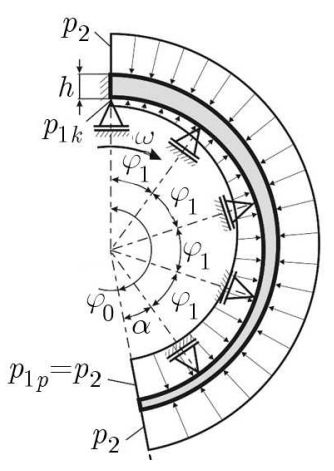

(b)

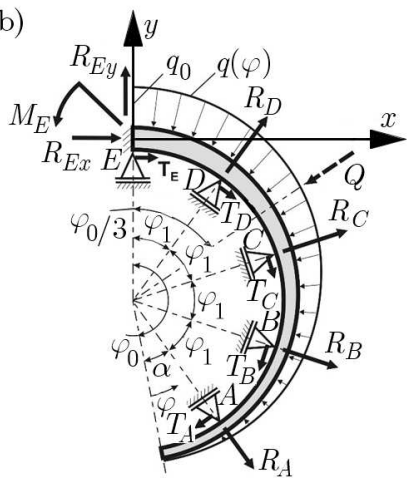

Fig. 6. (a) Static diagram of the beam with the wrapping angle $\varphi_{0}=169^{\circ}$, loaded with working pressure $p_{1}$ and compensating pressure $p_{2}$, (b) diagram after introduction of continuous load replacing the action of pressures $p_{1}$ and $p_{2}$

In this case, the chamber beam is in contact with five teeth of the gear and the working pressure $p_{1}$ decreases from the initial value $p_{1 p}=p_{2}$ for $\varphi=0$ down to the end value $p_{1 k}=0.166 p_{2}$ for $\varphi=\varphi_{0}$. Besides the continuous load, as shown in Fig. $4 \mathrm{~b}$, the friction forces $T_{A}, T_{B}, T_{C}, T_{D}, T_{E}$, reaction forces in the supports $R_{A}, R_{B}, R_{C}, R_{D}$ and the forces in the beam fixing $R_{E x}, R_{E y}, M_{E}$ act on the beam. 


\subsubsection{Solutions for the beam subject to load static scheme}

The static equilibrium equations for the considered beam assume the form

$$
\begin{aligned}
& \sum P_{x}=R_{E x}+R_{D x}+R_{C x}+R_{B x}+R_{A x}+T_{E}+T_{D x}+T_{C x}-T_{B x}-T_{A x}-Q_{x}=0 \\
& \sum P_{y}=R_{E y}+R_{D y}+R_{C y}-R_{B y}-R_{A y}-T_{D y}-T_{C y}-T_{B y}-T_{A y}-Q_{y}=0 \\
& \sum M^{E}=M_{E}+R_{D} r \sin \varphi_{1}+R_{C} r \sin 2 \varphi_{1}+R_{B} r \cos \frac{\varphi_{1}}{2}+R_{A} r \cos \frac{3 \varphi_{1}}{2} \\
& \quad-T_{D}\left(r-r \cos \varphi_{1}\right)-T_{C}\left(r-r \cos 2 \varphi_{1}\right)-T_{B}\left(r+r \sin \frac{\varphi_{1}}{2}\right) \\
& \quad-T_{A}\left(r+r \sin \frac{3 \varphi_{1}}{2}\right)-Q r \sin \frac{\varphi_{c}}{3}=0
\end{aligned}
$$

The reaction and friction force components in equations (3.13) are described by the formulas: $R_{A x}=R_{A} \cos \left(3 \varphi_{1} / 2\right), R_{A y}=R_{A} \sin \left(3 \varphi_{1} / 2\right), R_{B x}=R_{B} \cos \left(\varphi_{1} / 2\right), R_{B y}=R_{B} \sin \left(\varphi_{1} / 2\right)$, $R_{C x}=R_{C} \sin 2 \varphi_{1}, R_{C y}=R_{C} \cos 2 \varphi_{1}, R_{D x}=R_{D} \sin \varphi_{1}, R_{D y}=R_{D} \cos \varphi_{1}, T_{A x}=T_{A} \sin \left(3 \varphi_{1} / 2\right)$, $T_{A y}=T_{A} \cos \left(3 \varphi_{1} / 2\right), T_{B x}=T_{B} \sin \left(\varphi_{1} / 2\right), T_{B y}=T_{B} \cos \left(\varphi_{1} / 2\right), T_{C x}=T_{C} \cos 2 \varphi_{1}$, $T_{C y}=T_{C} \sin 2 \varphi_{1}, T_{D x}=T_{D} \cos \varphi_{1}, T_{D y}=T_{D} \sin \varphi_{1}, Q_{x}=Q \sin \left(\varphi_{0} / 3\right), Q_{y}=Q \cos \left(\varphi_{0} / 3\right)$.

In order to determine the four hyperstatic reactions (assumed here as the reactions in the supports) one should formulate four Menabrei equations

$$
\begin{aligned}
& \frac{\partial V}{\partial R_{A}}=\frac{1}{E I}\left[\int_{\alpha}^{\alpha_{2}}\left(-\frac{q_{0} r^{2} \varphi^{2}}{2 \varphi_{0}} \sin \frac{\varphi}{3}+R_{A} r \sin (\varphi-\alpha)-T_{A} r[1-\cos (\varphi-\alpha)]\right)[r \sin (\varphi-\alpha)] d \varphi\right] \\
& \quad+\frac{1}{E I}\left[\int_{\alpha_{2}}^{\alpha_{3}}-\frac{q_{0} r^{2} \varphi^{2}}{2 \varphi_{0}} \sin \frac{\varphi}{3}+R_{A} r \sin (\varphi-\alpha)-T_{A} r[1-\cos (\varphi-\alpha)]\right. \\
& \left.\left.\quad+R_{B} r \sin \left(\varphi-\alpha_{2}\right)-T_{B} r\left[1-\cos \left(\varphi-\alpha_{2}\right)\right]\right)[r \sin (\varphi-\alpha)] d \varphi\right] \\
& \quad+\frac{1}{E I}\left[\int _ { \alpha _ { 3 } } ^ { \alpha _ { 4 } } \left(-\frac{q_{0} r^{2} \varphi^{2}}{2 \varphi_{0}} \sin \frac{\varphi}{3}+R_{A} r \sin (\varphi-\alpha)-T_{A} r[1-\cos (\varphi-\alpha)]+R_{B} r \sin \left(\varphi-\alpha_{2}\right)\right.\right. \\
& \left.\left.\quad-T_{B} r\left[1-\cos \left(\varphi-\alpha_{2}\right)\right]+R_{C} r \sin \left(\varphi-\alpha_{3}\right)-T_{C} r\left[1-\cos \left(\varphi-\alpha_{3}\right)\right]\right)[r \sin (\varphi-\alpha)] d \varphi\right] \\
& \quad+\frac{1}{E I}\left[\int _ { \alpha _ { 4 } } ^ { \varphi _ { 0 } } \left(-\frac{q_{0} r^{2} \varphi^{2}}{2 \varphi_{0}} \sin \frac{\varphi}{3}+R_{A} r \sin (\varphi-\alpha)-T_{A} r[1-\cos (\varphi-\alpha)]\right.\right. \\
& \quad+R_{B} r \sin \left(\varphi-\alpha_{2}\right)-T_{B} r\left[1-\cos \left(\varphi-\alpha_{2}\right)\right]+R_{C} r \sin \left(\varphi-\alpha_{3}\right)-T_{C} r\left[1-\cos \left(\varphi-\alpha_{3}\right)\right] \\
& \left.\left.\quad+R_{D} r \sin \left(\varphi-\alpha_{4}\right)-T_{D} r\left[1-\cos \left(\varphi-\alpha_{4}\right)\right]\right)[r \sin (\varphi-\alpha)] d \varphi\right]=0 \\
& \quad+\frac{1}{E I}\left[\int _ { \alpha _ { 3 } } ^ { \alpha _ { 4 } } \left(-\frac{q_{0} r^{2} \varphi^{2}}{2 \varphi_{0}} \sin \frac{\varphi}{3}+R_{A} r \sin (\varphi-\alpha)-T_{A} r[1-\cos (\varphi-\alpha)]+R_{B} r \sin \left(\varphi-\alpha_{2}\right)\right.\right. \\
& \left.\left.\quad R_{B} r \sin \left(\varphi-\alpha_{2}\right)-T_{B} r\left[1-\cos \left(\varphi-\alpha_{2}\right)\right]\right)\left[r \sin \left(\varphi-\alpha_{2}\right)\right] d \varphi\right] \\
& \frac{1}{E I}\left[\int _ { \alpha _ { 2 } } ^ { \alpha _ { 3 } } \left(-\frac{q_{0} r^{2} \varphi^{2}}{2 \varphi_{0}} \sin \frac{\varphi}{3}+R_{A} r \sin (\varphi-\alpha)-T_{A} r[1-\cos (\varphi-\alpha)]\right.\right.
\end{aligned}
$$




$$
\begin{aligned}
& \left.\left.-T_{B} r\left[1-\cos \left(\varphi-\alpha_{2}\right)\right]+R_{C} r \sin \left(\varphi-\alpha_{3}\right)-T_{C} r\left[1-\cos \left(\varphi-\alpha_{3}\right)\right]\right)\left[r \sin \left(\varphi-\alpha_{2}\right)\right] d \varphi\right] \\
& +\frac{1}{E I}\left[\int _ { \alpha _ { 4 } } ^ { \varphi _ { 0 } } \left(-\frac{q_{0} r^{2} \varphi^{2}}{2 \varphi_{0}} \sin \frac{\varphi}{3}+R_{A} r \sin (\varphi-\alpha)-T_{A} r[1-\cos (\varphi-\alpha)]\right.\right. \\
& +R_{B} r \sin \left(\varphi-\alpha_{2}\right)-T_{B} r\left[1-\cos \left(\varphi-\alpha_{2}\right)\right]+R_{C} r \sin \left(\varphi-\alpha_{3}\right)-T_{C} r\left[1-\cos \left(\varphi-\alpha_{3}\right)\right] \\
& \left.\left.+R_{D} r \sin \left(\varphi-\alpha_{4}\right)-T_{C} r\left[1-\cos \left(\varphi-\alpha_{4}\right)\right]\right)\left[r \sin \left(\varphi-\alpha_{2}\right)\right] d \varphi\right]=0 \\
& \frac{\partial V}{\partial R_{C}}=\frac{1}{E I}\left[\int _ { \alpha _ { 3 } } ^ { \alpha _ { 4 } } \left(-\frac{q_{0} r^{2} \varphi^{2}}{2 \varphi_{0}} \sin \frac{\varphi}{3}+R_{A} r \sin (\varphi-\alpha)-T_{A} r[1-\cos (\varphi-\alpha)]\right.\right. \\
& +R_{B} r \sin \left(\varphi-\alpha_{2}\right)-T_{B} r\left[1-\cos \left(\varphi-\alpha_{2}\right)\right]+R_{C} r \sin \left(\varphi-\alpha_{3}\right) \\
& \left.\left.-T_{C} r\left[1-\cos \left(\varphi-\alpha_{3}\right)\right]\right)\left[r \sin \left(\varphi-\alpha_{3}\right)\right] d \varphi\right] \\
& +\frac{1}{E I}\left[\int _ { \alpha _ { 4 } } ^ { \varphi _ { 0 } } \left(-\frac{q_{0} r^{2} \varphi^{2}}{2 \varphi_{0}} \sin \frac{\varphi}{3}+R_{A} r \sin (\varphi-\alpha)-T_{A} r[1-\cos (\varphi-\alpha)]\right.\right. \\
& +R_{B} r \sin \left(\varphi-\alpha_{2}\right)-T_{B} r\left[1-\cos \left(\varphi-\alpha_{2}\right)\right]+R_{C} r \sin \left(\varphi-\alpha_{3}\right)-T_{C} r\left[1-\cos \left(\varphi-\alpha_{3}\right)\right] \\
& \left.\left.+R_{D} r \sin \left(\varphi-\alpha_{4}\right)-T_{D} r\left[1-\cos \left(\varphi-\alpha_{4}\right)\right]\right)\left[r \sin \left(\varphi-\alpha_{3}\right)\right] d \varphi\right]=0 \\
& \frac{\partial V}{\partial R_{D}}=\frac{1}{E I}\left[\int _ { \alpha _ { 4 } } ^ { \varphi _ { 0 } } \left(-\frac{q_{0} r^{2} \varphi^{2}}{2 \varphi_{0}} \sin \frac{\varphi}{3}+R_{A} r \sin (\varphi-\alpha)-T_{A} r[1-\cos (\varphi-\alpha)]\right.\right. \\
& +R_{B} r \sin \left(\varphi-\alpha_{2}\right)-T_{B} r\left[1-\cos \left(\varphi-\alpha_{2}\right)\right]+R_{C} r \sin \left(\varphi-\alpha_{3}\right)-T_{C} r\left[1-\cos \left(\varphi-\alpha_{3}\right)\right] \\
& \left.\left.+R_{D} r \sin \left(\varphi-\alpha_{4}\right)-T_{C D} r\left[1-\cos \left(\varphi-\alpha_{4}\right)\right]\right)\left[r \sin \left(\varphi-\alpha_{4}\right)\right] d \varphi\right]=0
\end{aligned}
$$

By solving the Menabrei equations one can determine reactions $R_{A}, R_{B}, R_{C}$ and $R_{D}$

$$
\begin{aligned}
& R_{A}=0.0908 \Delta p b r-0.4034 T_{A}-0.0255 T_{B}+0.0073 T_{C}-0.0019 T_{D}=1548.2 \mathrm{~N} \\
& R_{B}=0.2542 \Delta p b r+0.7128 T_{A}+0.3001 T B-0.0400 T_{C}+0.0104 T_{D}=4352.4 \mathrm{~N} \\
& R_{C}=0.4593 \Delta p b r+0.6065 T_{A}+0.6777 T_{B}+0.3127 T_{C}-0.0400 T_{D}=7863.2 \mathrm{~N} \\
& R_{D}=0.7524 \Delta p b r+0.6340 T_{A}+0.6126 T_{B}+0.6750 T_{C}+0.3027 T_{D}=12880.2 \mathrm{~N}
\end{aligned}
$$
fixing

Finally, using static equilibrium equations (3.13), one can calculate reactions in the beam

$$
\begin{aligned}
R_{E x} & =Q_{x}-R_{D x}-R_{C x}-R_{B x}-R_{A x}-T_{E}-T_{D x}-T_{C x}-T_{B x}-T_{A x}=877.0 \mathrm{~N} \\
R_{E y} & =Q_{y}-R_{D y}-R_{C y}+R_{B y}+R_{A y}+T_{D y}+T_{C y}+T_{B y}+T_{A y}=3747.4 \mathrm{~N} \\
M_{E} & =Q r \sin \frac{\varphi_{0}}{3}-R_{D} r \sin \varphi_{1}-R_{C} r \sin 2 \varphi_{1}-R_{B} r \cos \frac{\varphi_{1}}{2}-R_{A} r \cos \frac{3 \varphi_{1}}{2} \\
& +T_{D}\left(r-r \cos \varphi_{1}\right)+T_{C}\left(r-r \cos 2 \varphi_{1}\right)+T_{B}\left(r+r \sin \frac{\varphi_{1}}{2}\right) \\
& +T_{A}\left(r+r \sin \frac{3 \varphi_{1}}{2}\right)=23.8 \mathrm{Nm}
\end{aligned}
$$




\section{Calculations of the minimal beam thickness in a fixed cross section}

The beam thickness $h$ must satisfy the strength condition for the fixed beam cross section. This cross section is loaded with reaction forces $R_{C}, R_{D}$ or $R_{E}$ for the pump geometric conditions at $\varphi_{0}$ respectively $102^{\circ}, 132^{\circ}, 169^{\circ}$ and the bending moment $M_{C}, M_{D}$ or $M_{E}$, respectively. The loads generate a complex state of stress in the fixed cross section. The shearing stress (produced by the tangential component of the reaction denoted generally as $R_{y}$ ) reaches the highest value in central fibres of the cross section while the bending stress reaches the highest value in extreme fibres.

The strength condition concerning the maximum shear stress has the form

$$
\tau_{\max }=\frac{3}{2} \frac{\left|R_{y}\right|}{b h} \leqslant k_{t}
$$

thus the minimal beam thickness must satisfy the criterion

$$
h_{\min (\tau)}=\frac{3}{2} \frac{\left|R_{y}\right|}{b k_{t}}
$$

The strength condition for the allowable normal stress must take into account the simultaneous action of the tensile (or compressive) stress produced by the reaction component $R_{x}$ and the bending stress generated by the fixing moment $M_{u}$ in the extreme fibres

$$
\sigma=\left|\sigma_{r}\right|+\left|\sigma_{g}\right| \leqslant k_{r}
$$

By substituting the normal stress values (calculated as for straight bars when beam curvature radius $r>6 h$, the error due to the shift of the neutral beam bending axis does not exceed $0.5 \%$ ) $\sigma_{r}=R_{x} /(b h), \sigma_{g}=6 M_{u} /\left(b h^{2}\right)$ into equation (4.3), one gets the following equation for the minimal beam thickness $h_{\text {min }}$

$$
b k_{r} h_{\text {min }}^{2}-\left|R_{x}\right| h_{\text {min }}-6\left|M_{u}\right|=0
$$

The solution of this equation yields the second value of the minimal beam thickness

$$
h_{\min (\tau)}=\frac{\left|R_{x}\right|+\sqrt{R_{x}^{2}+24 M_{u} b k_{r}}}{2 b k_{r}}
$$

One should adopt the second value of the two values obtained from formulas (4.2) and (4.5) as the minimal beam width $h$.

Table 1 shows exemplary minimal beam thickness values calculated for three geometric versions of the pump, assuming the experimental friction forces generated by teeth of the gears $T_{i}=7.96 \mathrm{~N}$, safety factor $n=1.4$ and permissible stresses: $k_{r}=350 \mathrm{MPa}$ and $k_{t}=400 \mathrm{MPa}$.

Table 1. Exemplary load values and the minimal thickness $h_{\min }$ for the beam with the wrapping angle $\varphi_{0}=102^{\circ}$

\begin{tabular}{|c|c|c|c|c|c|}
\hline $\begin{array}{c}\text { Beam wrapping } \\
\text { angle }\end{array}$ & $r$ & $b$ & $p_{1 k}$ & $p_{2}$ & $\boldsymbol{h}_{\text {min }}$ \\
\hline \hline$\varphi_{0}=102^{\circ}$ & $26 \mathrm{~mm}$ & $26.3 \mathrm{~mm}$ & $15 \mathrm{MPa}$ & $30 \mathrm{MPa}$ & $\mathbf{2 . 9 8} \mathbf{m m}$ \\
\hline$\varphi_{0}=132^{\circ}$ & $26 \mathrm{~mm}$ & $26.3 \mathrm{~mm}$ & $10 \mathrm{MPa}$ & $30 \mathrm{MPa}$ & $\mathbf{3 . 2 6} \mathbf{~ m m}$ \\
\hline$\varphi_{0}=169^{\circ}$ & $26 \mathrm{~mm}$ & $26.3 \mathrm{~mm}$ & $5 \mathrm{MPa}$ & $30 \mathrm{MPa}$ & $\mathbf{3 . 9 9} \mathbf{~ m m}$ \\
\hline
\end{tabular}

The results of the static strength calculations show that from among the three versions of the compensating chamber the most advantageous one is the version with the beam with thw wrapping angle $\varphi_{0}=102^{\circ}$ for which the minimal beam thickness amounts to $2.98 \mathrm{~mm}$. In the case of the other versions, the beam thickness needs to be increased: 
- for the beam with the wrapping angle $\varphi_{0}=132^{\circ}$, the minimal thickness amounts to $3.26 \mathrm{~mm}$ (an increase by $9.4 \%$ ),

- for the beam with the wrapping angle $\varphi_{0}=169^{\circ}$, the minimal thickness amounts to $3.99 \mathrm{~mm}$ (an increase by $31 \%$ ).

Experimental studies are planned to be carried out on prototypes of the pump in order to verify the results of the calculations.

\section{References}

1. Chrobot M., Łabik M., Siudy B., Wieczorek K., 1997, Hydraulic optimization of axial backlash compensation (in Polish), Report Series Report, No. S-019/97

2. Dyląg Z., Jakubowicz A., Oreoś Z., 1999, Strength of Materials (in Polish), Vol. I/II, WNT, Warsaw

3. Judin E.M., 1958, Gear Pumps (in Polish), PWT, Warsaw

4. KolleK W., 2004, Fundamental of the Design of Hydraulic Drives and Controls (in Polish), Wrocław University of Technology Publishing House, Wrocław

5. Kollek W., 1996, Gear Pumps - Design and Operation (in Polish), Ossolineum, Wrocław

6. Kollek W., Radziwanowska U., 2015, Energetic efficiency of gear micropumps, Archives of Civil and Mechanical Engineering, 15, 1, 109-115

7. Niezgodziński M. E., Niezgodziński T., 1996, Strength of Materials (in Polish), PWN, Warsaw

8. Osiński P., 2012a, An external gear pump (in Polish), Patent application No. P398436, Warsaw

9. Osiński P., 2012b, An external gear pump (in Polish), Patent application No. P398437, Warsaw

10. Osiński P., 2013, High-Pressure and Low-Fluctuation Internal Gear Pumps (in Polish) Wrocław University of Technology Publishing House, Wrocław

11. Osiński P., DeptuŁa A., Partyka M.A., 2013, Discrete optimization of a gear pump after tooth root undercutting by means of multi-valued logic trees, Archives of Civil and Mechanical Engineering, 13, 4, 422-431, http://dx.doi.org/10.1016/j.acme.2013.05.001

12. Osiński P., Kania A., Kollek W., Wiczkowski E., 2012, An external gear pump (in Polish), Patent application No. P397539, Warsaw

13. Osiński P., Kollek W., 2007, Modelling and Design of Gear Pumps, Wrocław University of Technology Publishing House, Wrocław

14. Ragunathan C., Manoharan C., 2012, Dynamic analysis of hydrodynamic gear pump performance using design of experiment stand operational parameters, IOSR Journal of Mechanical and Civil Engineering, 1, 6, 17-23

15. Singal R.K., Singal M., Singal R., 2009, Hydraulic Machines. Fluid Machinery, I.K. International Publishing House, New Delhi

16. StryczeK S., 1995, Hydrostatic Drives (in Polish), Vol. I, II. WNT, Warsaw

17. VaCCA A., Guidetti M., 2011, Modelling and experimental validation of external spur gear machines for fluid power applications, Simulation Modelling Practice and Theory, 19, 2007-2031

18. Wang S., Sakurai H., Kasarekar A., 2011, The optimal design in external gear pumps and motors, ASME Transactions on Mechatronics, 16, 5.

19. Wiczkowski E., 2012, An external gear pump (in Polish), Patent application No. P397540, Warsaw

20. Zakrzewski M., Zawadzki J., 1983, Strength of Materials (in Polish), PWN, Warsaw 